\title{
Untargeted lipidomics reveals differences in the lipid pattern among clinical isolates of Staphylococcus aureus resistant and sensitive to antibiotics.
}

Weronika Hewelt-Belka ${ }^{1 *}$, Joanna Nakonieczna ${ }^{2}$, Mariusz Belka ${ }^{3}$,Tomasz Baczek ${ }^{3}$, Jacek

Namieśnik ${ }^{1}$, Agata Kot-Wasik ${ }^{1}$

${ }^{1}$ Department of Analytical Chemistry, Faculty of Chemistry, Gdańsk University of Technology, Gabriela Narutowicza 11/12, 80-233 Gdańsk, Poland

${ }^{2}$ Department of Biotechnology, Intercollegiate Faculty of Biotechnology, University of Gdańsk and Medical University of Gdańsk, Kładki 24, 80-822 Gdańsk, Poland

${ }^{3}$ Department of Pharmaceutical Chemistry, Medical University of Gdańsk, Al. Gen. J. Hallera 107, 80-416 Gdańsk, Poland

*Corresponding author: e-mail, weronika.hb@gmail.com.

\section{Table of contents}

\begin{tabular}{|c|c|c|}
\hline & Supporting Information & Page \\
\hline \multicolumn{3}{|c|}{$\begin{array}{l}\text { Results and Discussion Section } \\
\end{array}$} \\
\hline Figure S-1 & $\begin{array}{l}\text { Representative extracted compound chromatograms obtained } \\
\text { from the LC-MS-Q-TOF analysis of crude lipid extracts of } S \text {. } \\
\text { aureus clinical isolates. }\end{array}$ & S-2 \\
\hline Table S-1 & $\begin{array}{l}\text { The identified lipid groups that significantly differed between } \\
\text { S. aureus isolates that were resistant and sensitive to selected } \\
\text { antibiotics. }\end{array}$ & S-6 \\
\hline Figure S-2 & $\begin{array}{l}\text { The MS/MS spectra acquired in positive ionization mode and } \\
\text { fragmentation patterns of the identified lipid classes. }\end{array}$ & S-8 \\
\hline
\end{tabular}




\section{Results and discussion section}

Figure S-1

(A) $\times 10^{6}$

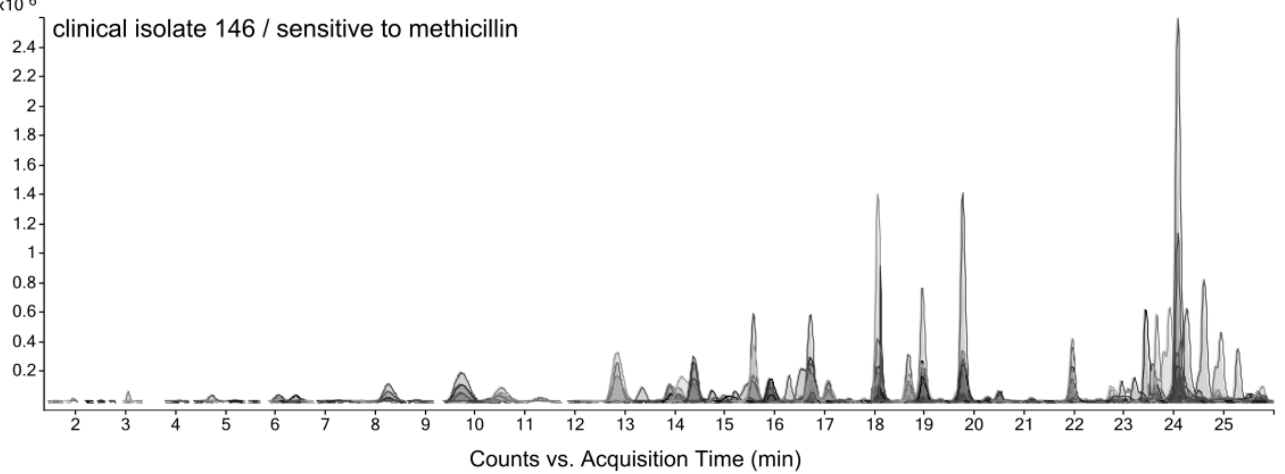

(B) $\times 10^{6}$

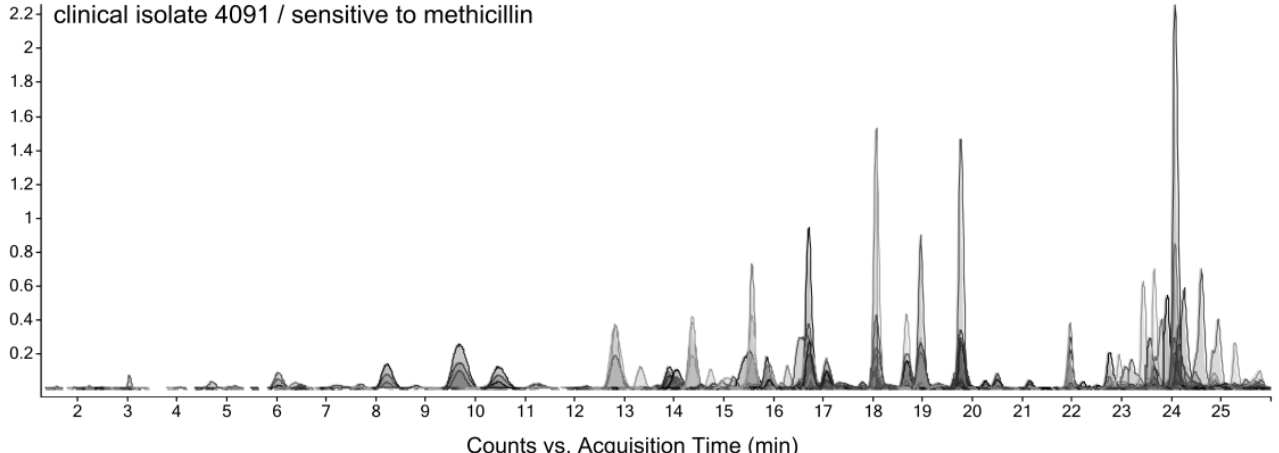

(C) $\times 10^{6}$

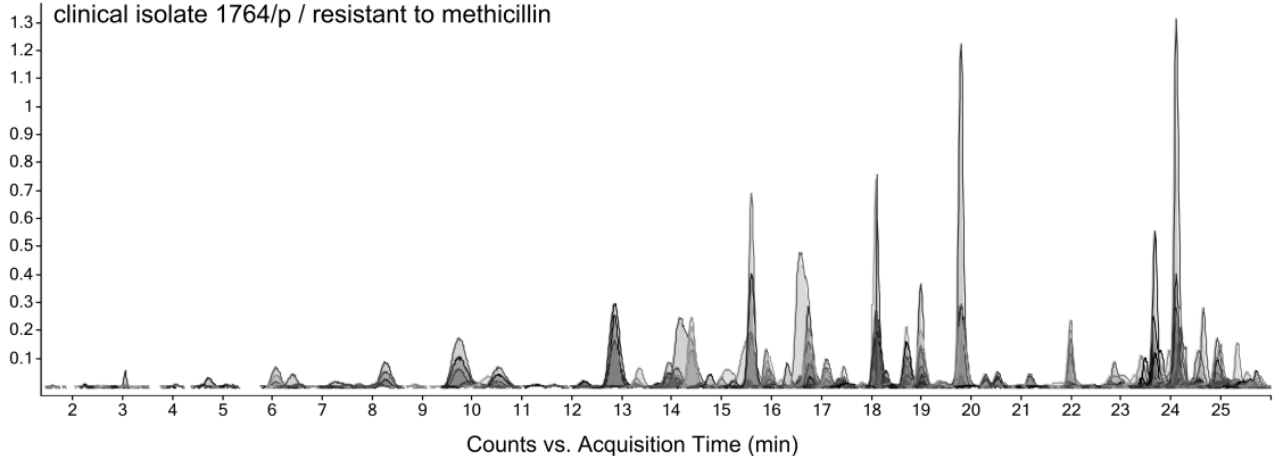

(D) $\times 10^{5}$

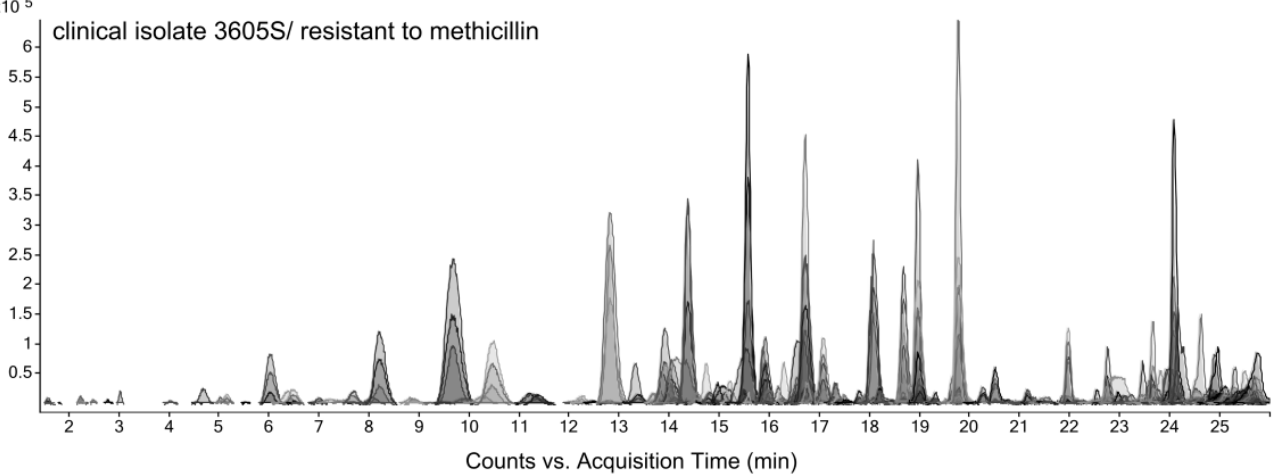


(E) $\times 10$

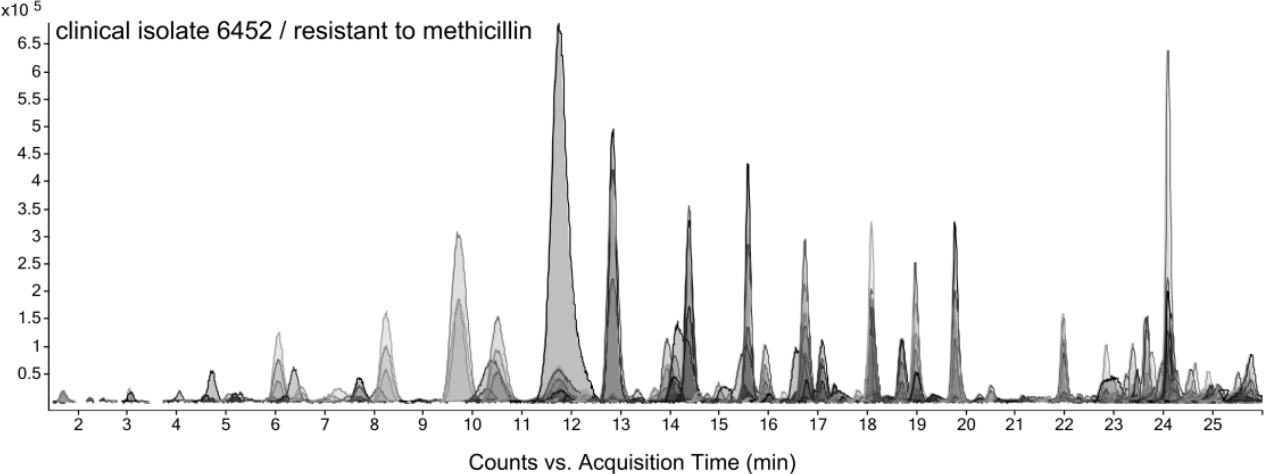

(F) $\times 10^{5}$

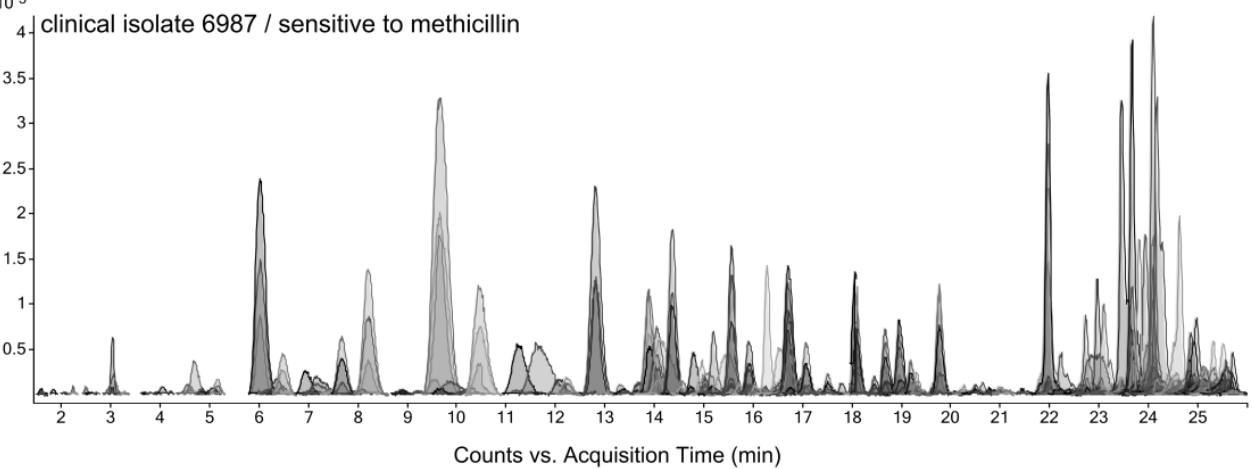

(G) $\times 10^{5}$

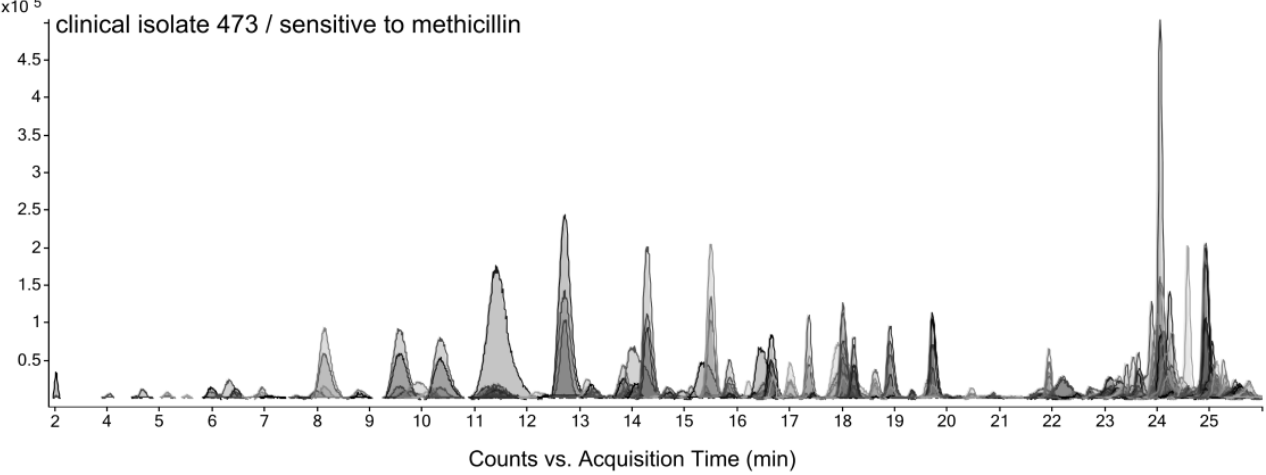

(H) $\times 10^{6}$

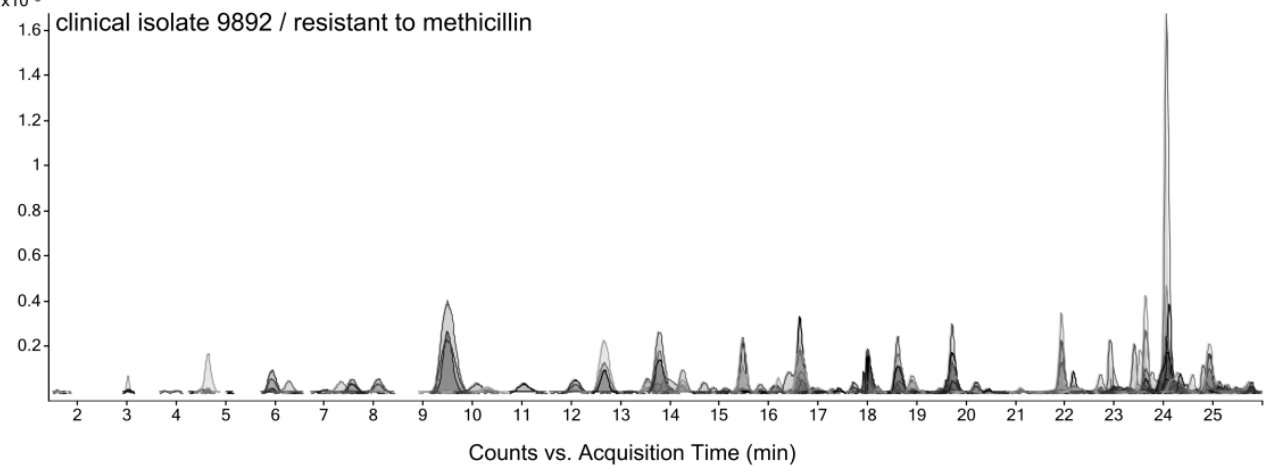



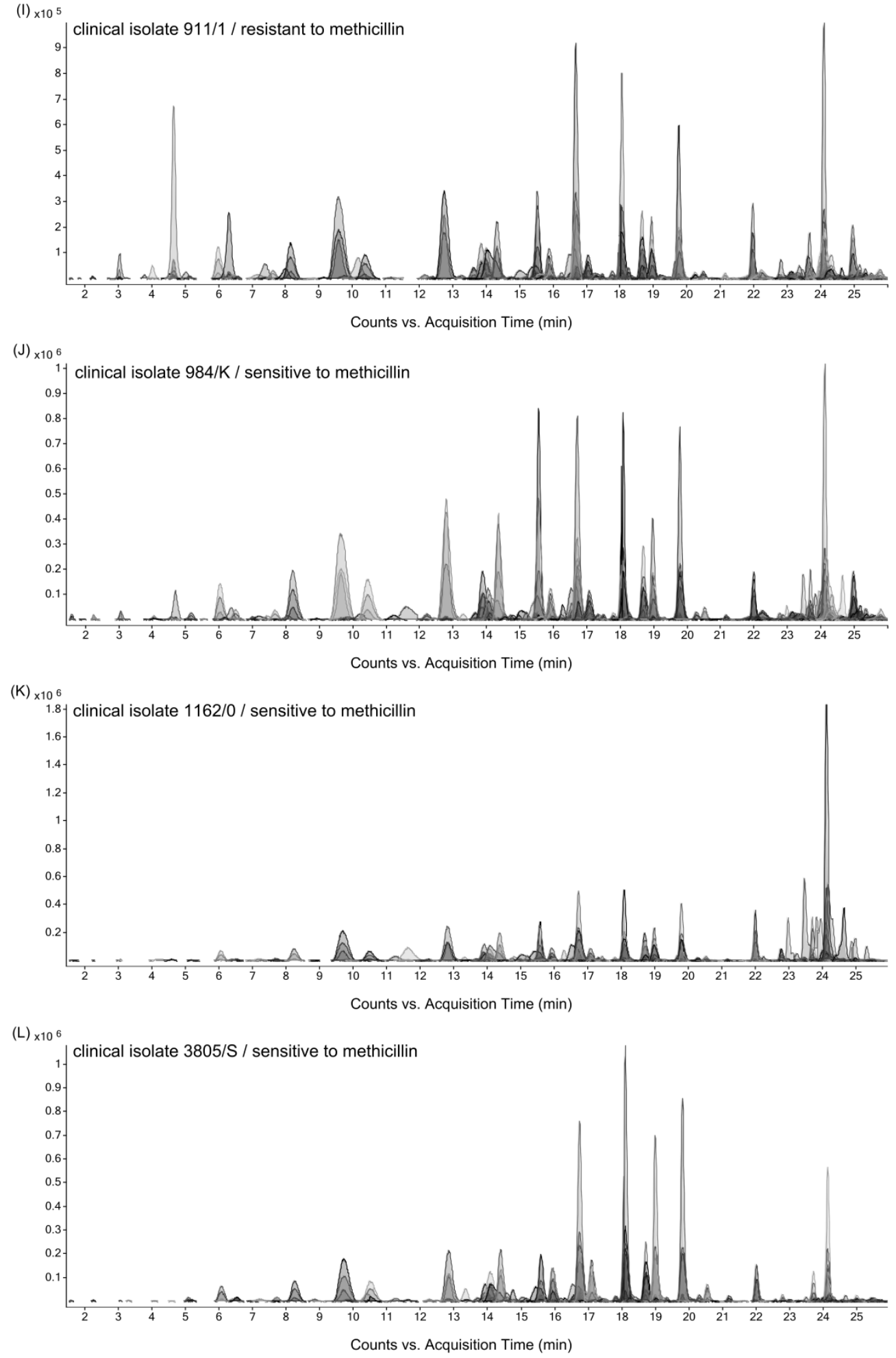

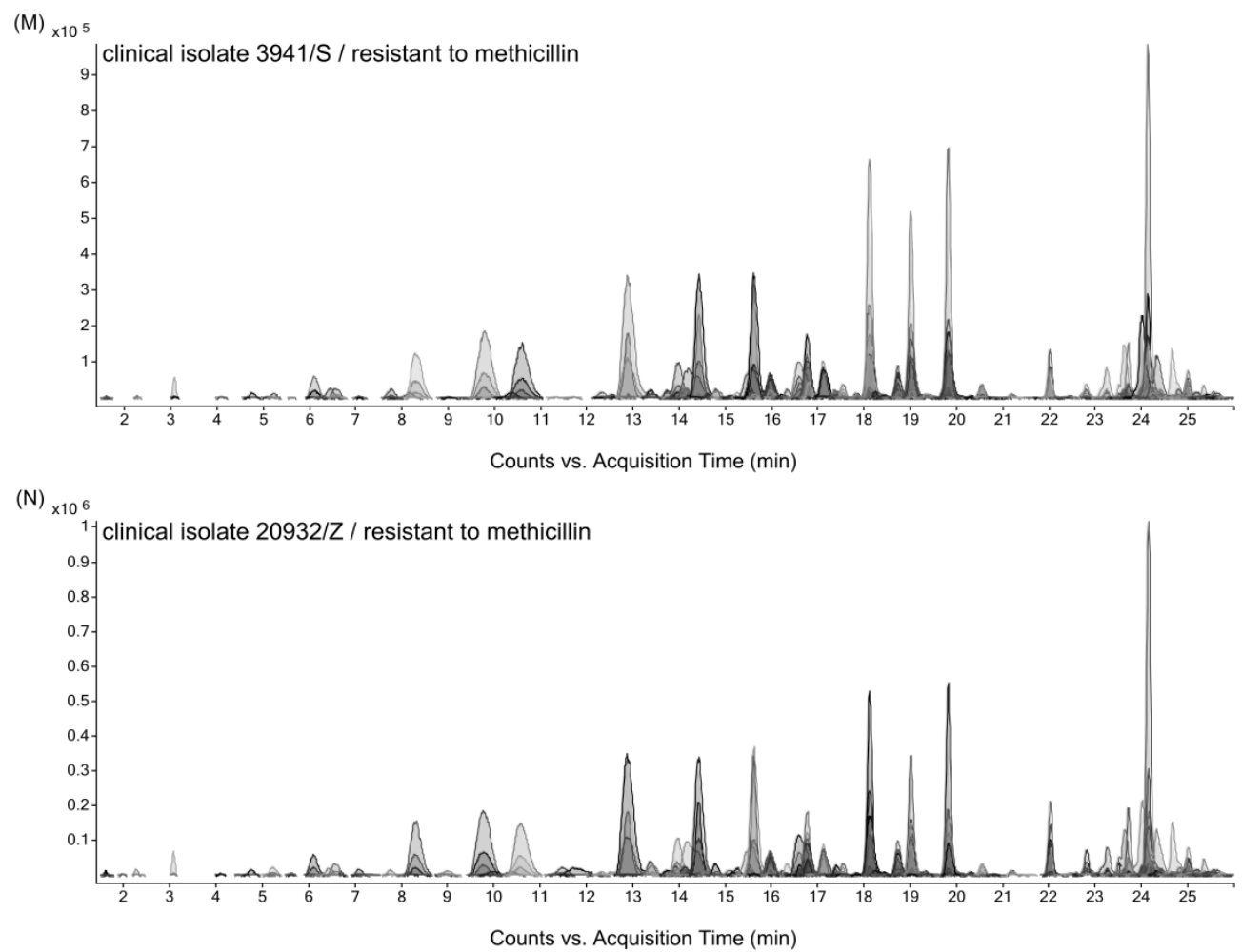

Figure S-1. Representative extracted compound chromatograms (ECCs) obtained from the LCMS-Q-TOF analysis of crude lipid extracts of $S$. aureus clinical isolates. 
Table S-1. The identified lipid groups that significantly differed between $S$. aureus isolates that were resistant and sensitive to selected antibiotics (please refer to Table 1 in the Results and Discussion section for results obtained for methicillin).

\begin{tabular}{|c|c|c|c|c|c|c|c|c|c|c|c|c|c|c|c|}
\hline \multirow[b]{2}{*}{ 号言 } & \multirow[b]{2}{*}{ 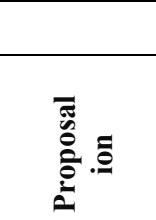 } & \multirow[b]{2}{*}{$\frac{\Xi}{\stackrel{\Xi}{\Xi}}$} & \multirow[b]{2}{*}{ 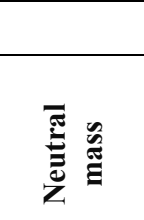 } & \multicolumn{3}{|c|}{ Gentamicin } & \multicolumn{3}{|c|}{ Ciprofloxacin } & \multicolumn{3}{|c|}{ Erythromycin } & \multicolumn{3}{|c|}{ Fusidic acid } \\
\hline & & & & 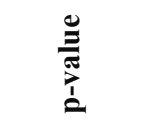 & $\hat{\nabla}$ & 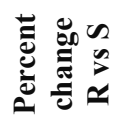 & 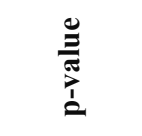 & $\hat{\nabla} \stackrel{0}{0}$ & 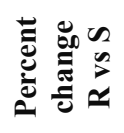 & 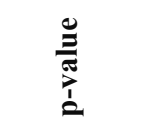 & $\hat{z} \stackrel{0}{0}$ & 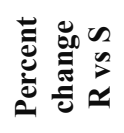 & 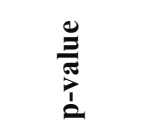 & $\Leftrightarrow \stackrel{0}{\partial}$ & 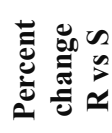 \\
\hline DG (30:0) & {$\left[\mathrm{M}+\mathrm{NH}_{4}\right]^{+}$} & 14.03 & 557.5017 & ns & 0.68 & -15.61 & 4.99E-04 & 1.28 & -27.62 & 1.44E-02 & 0.91 & -19.85 & $\mathrm{~ns}$ & 0.02 & -5.35 \\
\hline DG (30:0) & {$[\mathrm{M}+\mathrm{K}]^{+}$} & 14.54 & 578.4309 & ns & 0.77 & -7.88 & ns & 1.08 & -17.33 & ns & 0.96 & -20.27 & ns & 1.13 & -25.50 \\
\hline DG (31:0) & {$\left[\mathrm{M}+\mathrm{NH}_{4}\right]^{+}$} & 15.54 & 571.5172 & ns & 0.03 & 5.42 & ns & 0.38 & -6.66 & ns & 0.19 & -3.25 & $7.21 \mathrm{E}-05$ & 0.90 & 34.82 \\
\hline DG (31:0) & {$[\mathrm{M}+\mathrm{K}]^{+}$} & 15.90 & 592.4468 & ns & 0.29 & -5.44 & ns & 0.54 & -7.96 & $3.15 \mathrm{E}-02$ & 0.91 & -15.24 & ns & 0.81 & -17.69 \\
\hline DG (32:0) & {$[\mathrm{M}+\mathrm{K}]^{+}$} & 17.06 & 606.4624 & $\mathrm{~ns}$ & 0.60 & -13.38 & $\mathrm{~ns}$ & 0.75 & -13.12 & ns & 0.73 & -14.94 & $8.83 \mathrm{E}-03$ & 1.05 & -27.30 \\
\hline DG (34:0) & {$\left[\mathrm{M}+\mathrm{NH}_{4}\right]^{+}$} & 18.95 & 613.5645 & $5.27 \mathrm{E}-09$ & 1.77 & -48.27 & $2.88 \mathrm{E}-04$ & 1.29 & -28.99 & $7.80 \mathrm{E}-03$ & 0.82 & -22.34 & $7.50 \mathrm{E}-08$ & 1.50 & -51.10 \\
\hline DG (35:0) & {$\left[\mathrm{M}+\mathrm{NH}_{4}\right]^{+}$} & 19.75 & 627.5802 & $1.90 \mathrm{E}-08$ & 1.75 & -41.70 & $2.78 \mathrm{E}-02$ & 0.62 & -12.86 & $4.53 \mathrm{E}-05$ & 1.17 & -26.53 & $1.32 \mathrm{E}-04$ & 1.09 & -33.04 \\
\hline DG (36:0) & {$\left[\mathrm{M}+\mathrm{NH}_{4}\right]^{+}$} & 20.49 & 641.5954 & $4.11 \mathrm{E}-09$ & 1.88 & -45.12 & $5.93 \mathrm{E}-05$ & 1.41 & -27.67 & $9.29 \mathrm{E}-05$ & 1.23 & -28.09 & $3.71 \mathrm{E}-07$ & 1.52 & -45.54 \\
\hline DG (37:0) & {$[\mathrm{M}+\mathrm{Na}]^{+}$} & 21.14 & 660.5660 & $1.05 \mathrm{E}-03$ & 1.04 & -29.12 & ns & 0.10 & 0.88 & $2.68 \mathrm{E}-03$ & 0.79 & -23.44 & ns & 0.27 & -14.34 \\
\hline DGDG (29:0) & {$[\mathrm{M}+\mathrm{Na}]_{+}$} & 5.16 & 872.5469 & $\mathrm{~ns}$ & 0.62 & -14.74 & ns & 0.93 & -20.82 & $2.03 \mathrm{E}-06$ & 1.04 & 54.03 & ns & 0.95 & -31.85 \\
\hline DGDG (30:0) & {$[\mathrm{M}+\mathrm{Na}]^{+}$} & 6.03 & 886.5628 & $5.66 \mathrm{E}-03$ & 0.21 & 9.98 & ns & 0.19 & -6.99 & $1.84 \mathrm{E}-03$ & 0.10 & 4.61 & $2.36 \mathrm{E}-08$ & 0.72 & 44.87 \\
\hline DGDG (30:0) & {$[\mathrm{M}+\mathrm{Na}]^{+}$} & 6.49 & 886.5627 & $\mathrm{~ns}$ & 0.32 & 2.69 & ns & 0.35 & -2.35 & $1.26 \mathrm{E}-06$ & 0.80 & 48.93 & $\mathrm{~ns}$ & 0.78 & -18.42 \\
\hline DGDG (31:0) & {$[\mathrm{M}+\mathrm{Na}]^{+}$} & 8.21 & 900.5786 & $\mathrm{~ns}$ & 0.60 & 16.00 & ns & 0.50 & 10.69 & $1.27 \mathrm{E}-06$ & 1.73 & 54.14 & ns & 0.13 & -4.05 \\
\hline DGDG (31:0) & {$[\mathrm{M}+\mathrm{Na}]^{+}$} & 7.67 & 900.5783 & $2.32 \mathrm{E}-03$ & 0.99 & 68.27 & $3.21 \mathrm{E}-02$ & 0.66 & 35.68 & $5.78 \mathrm{E}-05$ & 0.71 & 49.92 & $8.16 \mathrm{E}-09$ & 1.43 & 127.26 \\
\hline DGDG $(32: 0)$ & {$\left[\mathrm{M}+\mathrm{NH}_{4}\right]^{+}$} & 9.66 & 909.6387 & $2.02 \mathrm{E}-03$ & 1.50 & 100.63 & ns & 0.87 & 43.26 & ns & 0.76 & 47.12 & $5.08 \mathrm{E}-09$ & 1.95 & 166.20 \\
\hline DGDG (32:0) & {$[\mathrm{M}+\mathrm{Na}]^{+}$} & 10.46 & 914.5942 & ns & 0.20 & 6.96 & ns & 0.48 & 14.14 & $2.04 \mathrm{E}-06$ & 1.48 & 64.98 & $\mathrm{~ns}$ & 0.54 & -21.30 \\
\hline DGDG (33:0) & {$[\mathrm{M}+\mathrm{Na}]^{+}$} & 12.22 & 928.6095 & $2.75 \mathrm{E}-03$ & 1.34 & 115.46 & $2.01 \mathrm{E}-02$ & 1.17 & 82.75 & $1.00 \mathrm{E}-06$ & 1.15 & 111.97 & $1.79 \mathrm{E}-07$ & 1.69 & 183.28 \\
\hline DGDG (33:0) & {$[\mathrm{M}+\mathrm{Na}]^{+}$} & 12.79 & 928.6100 & $1.47 \mathrm{E}-03$ & 1.06 & 24.09 & $9.57 \mathrm{E}-04$ & 1.35 & 25.77 & $3.14 \mathrm{E}-11$ & 2.05 & 53.37 & $\mathrm{~ns}$ & 0.12 & 2.97 \\
\hline DGDG (34:0) & {$[\mathrm{M}+\mathrm{Na}]^{+}$} & 13.88 & 942.6255 & $4.67 \mathrm{E}-03$ & 1.31 & 54.53 & $\mathrm{~ns}$ & 0.89 & 29.09 & $5.58 \mathrm{E}-06$ & 1.20 & 52.68 & $1.47 \mathrm{E}-05$ & 1.59 & 81.09 \\
\hline DGDG (35:0) & {$[\mathrm{M}+\mathrm{Na}]^{+}$} & 15.54 & 956.6414 & $\mathrm{~ns}$ & 0.07 & 1.38 & $\mathrm{~ns}$ & 0.74 & 13.19 & $5.58 \mathrm{E}-06$ & 1.55 & 36.95 & $2.41 \mathrm{E}-02$ & 0.71 & -17.14 \\
\hline Lys-PG (30:0) & {$[\mathrm{M}+\mathrm{H}]^{+}$} & 7.21 & 822.5730 & $1.23 \mathrm{E}-03$ & 1.38 & 54.12 & $6.66 \mathrm{E}-03$ & 1.29 & 42.66 & $\mathrm{~ns}$ & 0.07 & 2.50 & $3.78 \mathrm{E}-07$ & 1.68 & 88.44 \\
\hline MGDG (31:0) & {$[\mathrm{M}+\mathrm{Na}]^{+}$} & 12.44 & 738.5255 & ns & 0.35 & 11.60 & $3.66 \mathrm{E}-03$ & 1.07 & 34.03 & $1.79 \mathrm{E}-08$ & 1.81 & 91.00 & ns & 0.08 & 1.78 \\
\hline MGDG (32:0) & {$[\mathrm{M}+\mathrm{Na}]^{+}$} & 13.66 & 752.5411 & $7.61 \mathrm{E}-04$ & 1.50 & 110.81 & $6.33 \mathrm{E}-04$ & 1.47 & 93.79 & $3.30 \mathrm{E}-08$ & 1.40 & 122.82 & $1.46 \mathrm{E}-09$ & 1.94 & 182.59 \\
\hline MGDG (32:0) & {$[\mathrm{M}+\mathrm{Na}]^{+}$} & 14.16 & 752.5411 & ns & 0.03 & 4.03 & $1.72 \mathrm{E}-02$ & 1.00 & 42.42 & $3.39 \mathrm{E}-06$ & 1.45 & 87.41 & ns & 0.30 & -11.34 \\
\hline MGDG (33:0) & {$[\mathrm{M}+\mathrm{Na}]^{+}$} & 15.45 & 766.5569 & $3.29 \mathrm{E}-05$ & 0.89 & 25.32 & 1.64E-09 & 2.01 & 53.56 & $1.52 \mathrm{E}-10$ & 1.94 & 66.60 & $5.29 \mathrm{E}-03$ & 0.47 & 15.44 \\
\hline MGDG (35:0) & {$[\mathrm{M}+\mathrm{Na}]^{+}$} & 17.48 & 794.5881 & ns & 0.18 & -7.53 & $3.07 \mathrm{E}-03$ & 0.96 & 38.68 & $1.54 \mathrm{E}-08$ & 1.55 & 94.65 & ns & 0.11 & -5.50 \\
\hline PG (31:0) & {$[\mathrm{DAG}]^{+\mathrm{c}}$} & 4.05 & 536.4803 & $1.58 \mathrm{E}-06$ & 1.76 & 127.05 & $5.51 \mathrm{E}-07$ & 1.77 & 109.69 & $1.46 \mathrm{E}-04$ & 1.08 & 86.31 & $1.98 \mathrm{E}-09$ & 1.94 & 173.00 \\
\hline PG (32:0) & {$[\mathrm{DAG}]^{+}$} & 4.69 & 550.4960 & 7.34E-05 & 1.64 & 341.09 & $1.72 \mathrm{E}-02$ & 1.32 & 203.78 & $\mathrm{~ns}$ & 1.04 & 218.26 & $1.16 \mathrm{E}-08$ & 1.86 & 464.31 \\
\hline PG (33:0) & {$[\mathrm{DAG}]^{+}$} & 6.36 & 564.5115 & $1.48 \mathrm{E}-10$ & 2.01 & 202.20 & $9.18 \mathrm{E}-13$ & 2.02 & 189.92 & $1.05 \mathrm{E}-06$ & 1.30 & 133.42 & $2.23 \mathrm{E}-10$ & 2.06 & 238.12 \\
\hline PG (35:0) & {$[\mathrm{DAG}]^{+}$} & 10.25 & 592.5427 & $1.39 \mathrm{E}-08$ & 1.88 & 152.19 & $8.01 \mathrm{E}-13$ & 2.26 & 187.00 & $2.77 \mathrm{E}-06$ & 1.15 & 98.17 & $5.00 \mathrm{E}-09$ & 1.97 & 187.68 \\
\hline
\end{tabular}


DG, diacylglycerol; DGDG, diglycosyldiacylglycerol; MGDG; monoglycosyldiacylglycerol; PG, phosphatidylglycerol; Lys-PG, lysyl-phosphatidylglycerol; ns - not significant difference.

${ }^{a}$ p-value calculated using Mann-Whitney U test, $\mathrm{p}<0.05 ;{ }^{\mathrm{b}}$ percent change of S. aureus particular lipid content in antibioticresistant isolates vs $\mathrm{S}$. aureus antibiotic-sensitive isolates; ${ }^{\mathrm{c}}$ fragment ion 


\section{Figure S-2}

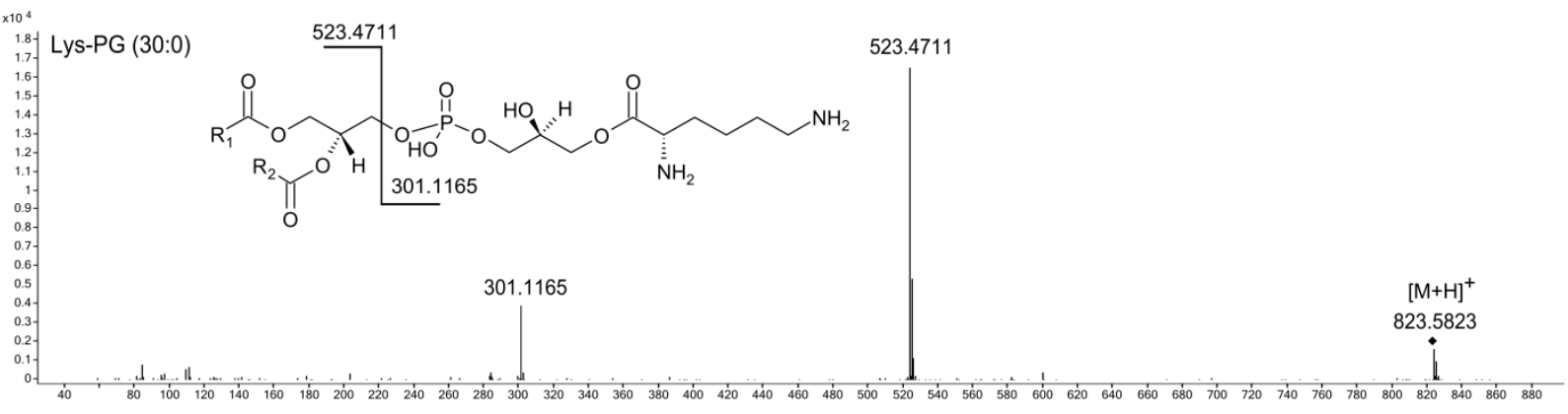

Counts vs. Mass-to-Charge (m/z)
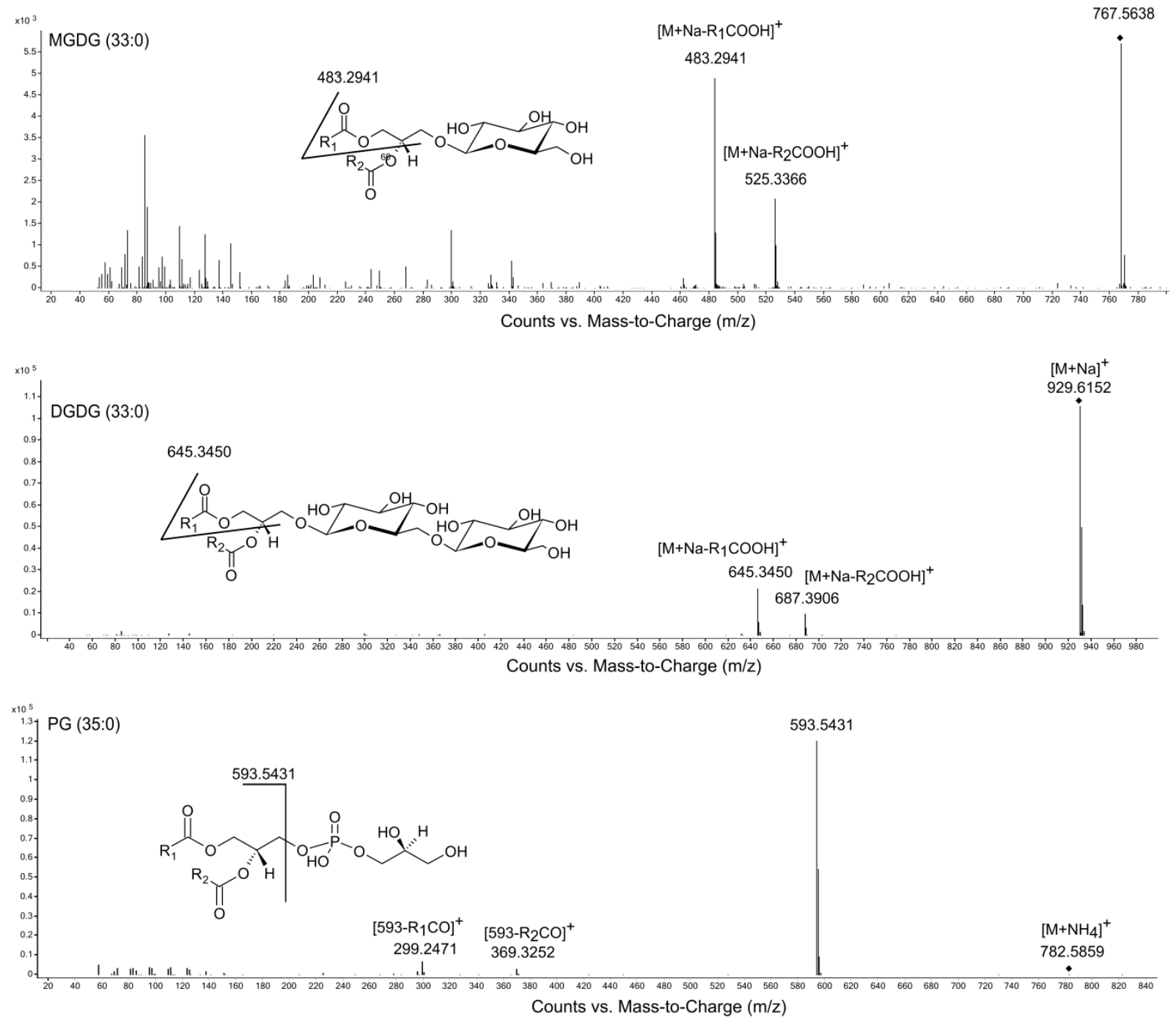


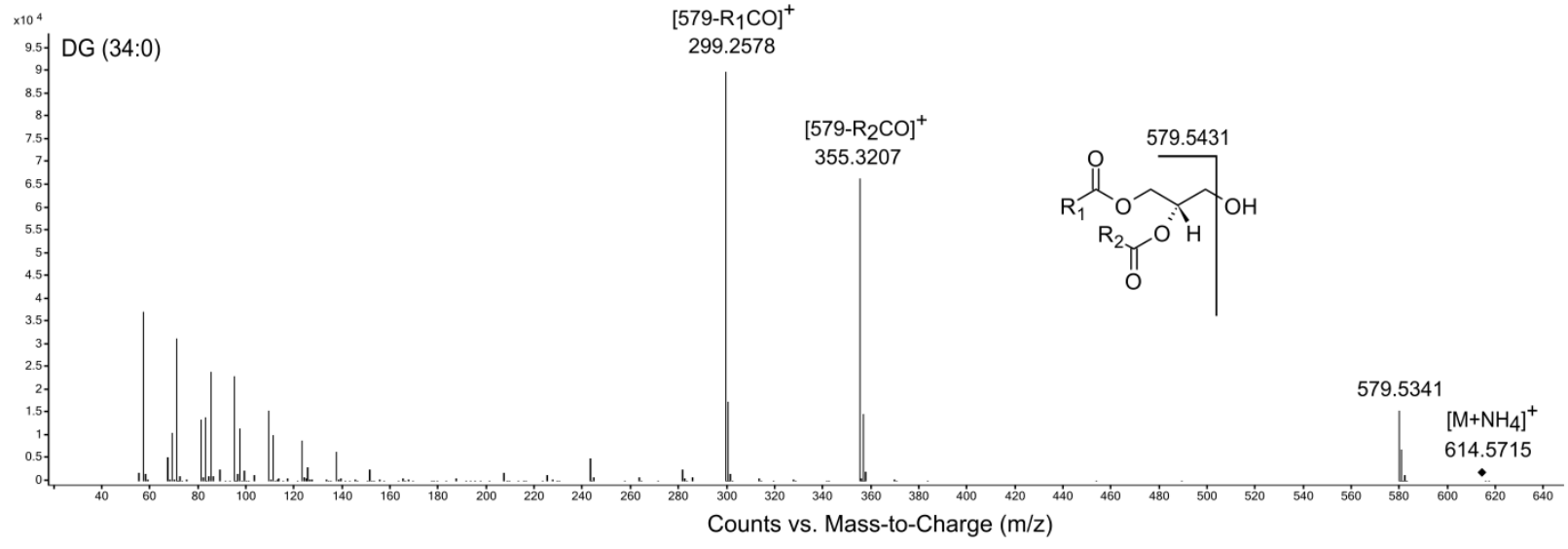

Figure S-2. The MS/MS spectra acquired in positive ionization mode and fragmentation patterns of the identified lipid classes. 